\title{
SUBALGEBRAS OF $B[c]$
}

\section{H. I. BROWN AND TAE-GEUN $\mathrm{CHO}^{1}$}

\begin{abstract}
Two classes of subalgebras of the bounded operators on the Banach space of convergent sequences are studied. One class contains the well-known algebra of conservative matrices and the other contains the algebra of almost matrices. It is shown that the nontrivial members within each class are isomorphic to each other.
\end{abstract}

We denote the Banach spaces of convergent and bounded complex sequences by $c$ and $m$, respectively, and the Banach algebra of bounded linear operators on $c$ (with the usual uniform norm) by $B[c]$. With lim denoting the functional $\lim x=\lim _{i} x_{i}$ on $c$ and with $e$ and $e^{k}$ denoting, respectively, the sequences $(1,1,1, \cdots)$ and $(0, \cdots, 0,1,0, \cdots)$, where 1 appears in the $k$ th coordinate and zeros elsewhere, $k=1,2, \cdots$, we have the important functionals $\chi$ and $\chi_{i}$ on $B[c]$ defined by (see [6, p. 241]) $\chi(T)=\lim (T e)-\sum_{k} \lim \left(T e^{k}\right)$ and $\chi_{i}(T)=(T e)_{i}-\sum_{k}\left(T e^{k}\right)_{i}, \quad i=1,2, \cdots$. (All sequence subscripts, as well as all indices of summation, run from 1 to $\infty$.)

As usual we identify $c^{* *}$ (the second dual space of $c$ ) with $m$. Then $T^{* *}$, the second adjoint of an operator $T$ in $B[c]$, is a mapping from $m$ to $m$ and has the following matrix representation:

$$
T^{* *}=\left[\begin{array}{ccccc}
\chi(T) & b_{1} & b_{2} & b_{3} & \cdots \\
\chi_{1}(T) & b_{11} & b_{12} & b_{13} & \cdots \\
\chi_{2}(T) & b_{21} & b_{22} & b_{23} & \cdots \\
\cdot & \cdot & \cdot & \cdot & \\
\cdot & \cdot & \cdot & \cdot & \\
\cdot & \cdot & \cdot & \cdot &
\end{array}\right]
$$

Received by the editors February 11, 1972 and, in revised form, October 27, 1972 and January 6, 1973.

AMS (MOS) subject classifications (1970). Primary 46A45, 46H10; Secondary 46A35, 47A05, 47B05.

Key words and phrases. Conservative matrix, conull matrix, adjoints, algebraic isomorphism, matrix algebras, subalgebras, summability, operators on Banach space, compact operators, scalar homomorphism.

${ }^{1}$ Clerical and publication costs partially supported by National Science Foundation Grant GU-3171. 
where $b_{n k}=\left(T e^{k}\right)_{n}$ and $b_{k}=\lim \left(T e^{k}\right)$. For further discussion of this representation and some of the remarks that follow, see, for example, [3] (and [1]). For each $T$ in $B[c]$ we have the unique representation $T=v \otimes \lim +B$, where $B$ is the matrix $\left(b_{n k}\right), v$ is the bounded sequence $\left(\chi_{i}(T)\right)$, and $v \otimes \lim$ denotes the one-dimensional operator from $c$ to $m$ which sends $x$ to $(\lim x) v$. Let

and let

$$
\Omega=\left\{T \in B[c]: \lim _{i} \chi_{i}(T) \text { exists }\right\}
$$

$$
\Gamma=\left\{T \in B[c]: \chi_{i}(T)=0 \text { for each } i\right\} .
$$

Then $\Omega$ and $\Gamma$ are proper subalgebras of $B[c]$ with $\Omega \supset \Gamma$. $\Gamma$ is the wellknown algebra of conservative matrices and $\chi$ is a scalar homomorphism on $\Gamma$. Those members $T$ in $\Gamma$ for which $\chi(T)=0$ are called conull and the algebra of conull matrices is denoted by $\Psi$. These and other subalgebras of $B[c]$ were studied in [1]. It was shown there that $\chi$ is the only nonzero scalar homomorphism on $\Gamma$, that $\Omega$ also supports only one nonzero scalar homomorphism denoted by $\rho$ and defined by $\rho(T)=\chi(B)$, and that $\Psi$, $\rho^{\perp}$ (the kernel of $\rho$ ), $\Gamma$, and $\Omega$ are the only proper subalgebras of $B[c]$ that contain $\Psi\left(=\rho^{\perp} \cap \Gamma\right)$.

More recently, A. Wilansky [5] introduced the following two classes of subalgebras of $B[c]$. (Our notation differs slightly from his so as to agree with the notation in [1].) Let $\hat{c}$ denote the canonical embedding of $c$ in $c^{* *}$. ( $\hat{c}$ is identified with the set of sequences converging to their first term. See, for example, $\left[7\right.$, p. 102].) Given any $w$ in $c^{* *}(=m)$ let

$$
\Gamma_{w}=\left\{T \in B[c]: T^{* *} w=\lambda w \text { for some scalar } \lambda\right\}
$$

and let

$$
\Omega_{w}=\left\{T \in B[c]: T^{* *} w \in w \oplus \hat{c}\right\},
$$

where by $w \oplus X$ ( $w$ a vector and $X$ a linear space) we mean the linear span $\{\lambda w+x: x \in X$ and $\lambda$ a scalar $\}$. Clearly, $\Gamma_{w}$ is contained in $\Omega_{w}$. If $w \notin \hat{c}$, then for $T \in \Omega_{w}$ (resp., $T \in \Gamma_{w}$ ), $T^{* *} w=\lambda w+\hat{x}$ (resp., $T^{* *} w^{*}=\lambda w$ ) thus defining a functional $\rho_{w}$ on $\Omega_{w}$ (resp., on $\Gamma_{w}$ ) by $\rho_{w}(T)=\lambda$. It is proved in $\left[5\right.$, p. 356] that $\Omega_{w}$ is a closed subalgebra of $B[c]$ containing the compact operators, that $\Gamma_{w}$ is a closed subalgebra of $\Omega_{w}$, and that $\rho_{w}$ is a scalar homorphism on $\Omega_{w}$ and on $\Gamma_{w}$ for $w^{\prime} \notin \hat{c}$. It is also shown that $\Omega_{w}=\Omega$, $\Gamma_{w}=\Gamma$, and $\rho_{w}=\rho$ when $w=e^{1}$.

In this paper we study these subalgebras in more detail. For example, we show that when $w \notin \hat{c}, \Omega_{w}$ (resp., $\Gamma_{w}$ ) is either $\Omega$ (resp., $\Gamma$ ) or is algebraically isomorphic with $\Omega$ (resp., $\Gamma$ ). It follows from this that all of the relationships between $B[c], \Omega, \rho^{\perp}$, and $\Gamma$ proved in [1] equally apply to $B[c], \Omega_{w}, \rho_{w}$, and $\Gamma_{w}$. We also show that $\bigcap\left\{\Gamma_{w}: w \in m\right\}=\{\lambda I: \lambda$ a scalar $\}$ 
and that $\bigcap\left\{\Omega_{w}: w \in m\right\}=I \oplus K$, where $I$ denotes the identity operator in $B[c]$ and $K$ denotes the set of all compact operators in $B[c]$.

We begin by listing some useful facts.

1. Lemma. (a) $\hat{c}$ may be identified with the set of sequences converging to their first term.

(b) $c=x \oplus \hat{c}$ for any convergent sequence $x$ which does not converge to its first term.

(c) If $z \notin w \oplus \hat{c}$ then $(w \oplus \hat{c}) \cap(z \oplus \hat{c})=\hat{c}$.

Proof. These statements are well known. Part (a), for example, is done in $[7$, p. 102].

2. LemMA. (a) If $w \notin \hat{c}$ and $z \in(w \oplus \hat{c}) \mid \hat{c}$ then $\Omega_{z}=\Omega_{w}$.

(b) If $z=\lambda w$ with $\lambda \neq 0$ then $\Gamma_{z}=\Gamma_{w}$.

Proof. In part (a) we have $z=\lambda w+\hat{x}$ with $\lambda \neq 0$ and $\hat{x} \in \hat{c}$. If $T \in \Omega_{z}$ then $T^{* *} z=\rho_{z}(T)_{z}+\hat{y}$ with $\hat{y} \in \hat{c}$, and so $T^{* *} w=(1 / \lambda) T^{* *}(z-\hat{x})=$ $(1 / \lambda)\left(\rho_{z}(T) z+\hat{y}-T^{* *} \hat{x}\right) \in w \oplus \hat{c}$. Hence, $\Omega_{z} \subset \Omega_{w}$. The reverse containment is proved similarly, as is part (b).

3. Lemma. (a) $w \in \hat{c}$ if and only if $\Omega_{w}=B[c]$.

(b) If $w \in \hat{c}$ then $\Gamma_{w} \neq \Gamma$.

Proof. The first half of (a) follows from the fact that $\hat{c}$ is invariant under every $T^{* *}$. To prove the second half assume $w \notin \hat{c}$. Then either $w$ converges with $\lim w \neq w_{1}$ (Lemma $\left.1(\mathrm{a})\right)$, or $w$ is divergent. In the first case let $v=(-1,0,-1,0, \cdots)$ and define $B=\left(b_{n k}\right)$ by setting $b_{2 n-1,2 n-1}=1$ $(n=1,2, \cdots)$ and $b_{n k}=0$ otherwise. Then $T=v \otimes \lim +B$ belongs to $B[c]$ and $T^{* *} w=\left(0, w_{2}-w_{1}, 0, w_{4}-w_{1}, 0, \cdots\right) \notin c$. Since $c=w \oplus \hat{c}$ (Lemma 1(b)), $T$ does not belong to $\Omega_{w}$. In the second case choose a subsequence $\left(w_{k(n)}\right)$ of $w$ (with $k(1)>1$ ) which converges to some number different from $w_{1}$, and let $T=\left(t_{n k}\right)$ be the subsequence selecting matrix defined by setting $t_{n, k-1}=1$ when $k=k(n)(n=1,2, \cdots)$ and 0 otherwise. Then $T \in B[c]$ and $T^{* *} w=\left(w_{1}, w_{k(1)}, w_{k(2)}, \cdots\right) \in c \mid \hat{c}$ and so $T^{* *} w$ does not belong to $w \oplus \hat{c}$. Hence we again have that $T \notin \Omega_{w}$.

To prove (b) simply observe that if $w$ is the zero sequence then $\Gamma_{w}=$ $B[c] \neq \Gamma$, while if $w \neq 0$ then $w^{\prime} \otimes \lim$ belongs to $\Gamma_{w}$ but does not belong to $\Gamma$, where $w^{\prime}=\left(w_{2}, w_{3}, w_{4}, \cdots\right)$.

The next lemma is Lemma 1.1 of [1]. We repeat it here for easy reference.

4. Lemma. Let $v$ and $x$ be bounded sequences with $v$ divergent. Then there exists a conull multiplicative matrix $B$ such that $B v=x$.

(A matrix $B$ in $\Gamma$ is conull and multiplicative if $\chi(B)=\chi_{i}(B)=$ $\lim \left(B e^{i}\right)=0$ for each $i=1,2, \cdots$.) 
5. THEOREM. Let $w \notin \hat{c}$. Then $\Omega_{z}=\Omega_{w}$ if and only if $z \in(w \oplus \hat{c}) \mid \hat{c}$.

Proof. The second half of the theorem is Lemma 2(a). To prove the first half assume that $z \notin(w \oplus \hat{c}) \mid \hat{c}$. If $z$ belongs to $\hat{c}$ use Lemma 3(a) to conclude that $\Omega_{z}=B[c] \neq \Omega_{w}$. If $z \notin \hat{c}$ then $z$ must have a cluster point $p$ different from $z_{1}$. By considering $z$-pe, if necessary, we assume that $z_{1} \neq 0$ and that $z$ has a subsequence $\left(z_{k(n)}\right)$ (with $\left.k(1)>1\right)$ which converges to 0 . (Notice that if $y=z-$ pe then $\Omega_{y}=\Omega_{z}$ by Lemma 2(a).) Now consider the corresponding subsequence $\left(w_{k(n)}\right)$ of $w$. It either converges or it diverges. In the latter case we may choose a subsequence $(m(n))$ of $(k(n))$ so that $\left(w_{m(n)}\right)$ converges. The subsequence $\left(z_{m(n)}\right)$ still converges to zero. Thus, without loss of generality we may assume that $\left(w_{k(n)}\right)$ converges. Moreover, by adding a constant sequence to $w$ (and using Lemma 2(a)) we may also assume that $\left(w_{k(n)}\right)$ converges to zero.

Let $T=\left(t_{n k}\right)$ be the subsequence selecting matrix $t_{n, k-1}=1$ if $k=k(n)$ $(n=1,2, \cdots)$; let $v$ be the bounded sequence $\left(1, w_{k(1)}, 1, w_{k(2)}, \cdots\right)$; define $B=\left(b_{n k}\right)$ by $b_{2 n-1,2 n-1}=-1(n=1,2, \cdots)$; and set $S=v \otimes l i m+B$. Then

$$
S^{* *}\left(T^{* *} w\right)=w_{1}\left(0,1, w_{k(1)}, 1, w_{k(2)}, \cdots\right)-\left(0, w_{k(1)}, 0, w_{k(3)}, \cdots\right)
$$

and

$$
S^{* *}\left(T^{* *} z\right)=z_{1}\left(0,1, w_{k(1)}, 1, w_{k(2)}, \cdots\right)-\left(0, z_{k(1)}, 0, z_{k(3)}, \cdots\right) .
$$

Now use Lemma 4 to get a conull multiplicative matrix $R$ such that $R v=$ $\left(w_{2}-w_{1}, w_{3}-w_{1}, w_{4}-w_{1}, \cdots\right)$. Then $(R S T)^{* *} w=w_{1} w-w_{1}^{2} e-R^{* *} \hat{y}$ and $(R S T)^{* *} z=z_{1} w-z_{1} w_{1} e-R^{* *} \hat{x}$, where $\hat{y}=\left(0, w_{k(1)}, 0, w_{k(3)}, \cdots\right) \in \hat{c}$ and $\hat{x}=\left(0, z_{k(1)}, 0, z_{k(3)}, \cdots\right) \in \hat{c}$. Thus both $(R S T)^{* *} w$ and $(R S T)^{* *} z$ belong to $w \oplus \hat{c}$. Since $z_{1} \neq 0,(R S T)^{* *} z$ does not belong to $\hat{c}$ and so it cannot belong to $z \oplus \hat{c}$ (by Lemma $1(\mathrm{c})$ ). Thus, $\Omega_{w} \neq \Omega_{z}$.

As an immediate consequence of Theorem 5, Lemma 1(b), and the fact that $\Omega=\Omega_{e^{1}}$ [5, Theorem 5] we get the following corollary.

\section{Corollary. $\Omega_{z}=\Omega$ if and only if $z \in c \mid \hat{c}$.}

In order to set up the algebra isomorphism from $B[c]$ onto $B[c]$ mentioned in the introduction we need to know that for each $w \notin \hat{c}$ we can produce an isomorphism in $B[c]$ whose second adjoint sends $w$ to $e^{1}$. We do this in two steps. First, we produce an isomorphism whose second adjoint sends $w$ to some $x$ in $c \mid \hat{c}$ (Lemma 7). Then we produce another isomorphism whose second adjoint sends $x$ to $e^{1}$ (Lemma 8). Our construction in Lemma 7 is patterned after the one in [2, Lemma 1].

7. Lemma. Let $w \notin \hat{c}$. Then there exists an isomorphism $T$ in $B[c]$ such that $T^{* *} w \in c \backslash \hat{c}$. 
Proof. Since $w$ is not in $\hat{c}$ we may choose a convergent subsequence $\left(w_{k(n)}\right)$ of $w$ (with $k(1)>1$ ) so that $w_{k(n)} \neq w_{1}$ for each $n$ and $\lim _{n} w_{k(n)}=$ $l \neq w_{1}$. Let $B=\left(b_{n k}\right)$ be defined as follows.

Set $b_{n n}=1$ for every $n$.

If $n=k(i)+j<k(i+1)-1$ for some $i=1,2,3, \cdots$, and some $j=$ $0,1,2, \cdots$, set $b_{n, k(i)-1}=\left(w_{n+1}-l\right) /\left(w_{1}-w_{k(i)}\right)$.

Let $b_{n k}=0$ for all other choices of $n$ and $k$. Next define $v$ as follows.

Set $v_{n}=0$ for $1 \leqq n<k(1)$ and for $n=k(i)-1, i=2,3,4, \cdots$.

If $n=k(i)+j<k(i+1)-1$ for some $i=1,2,3, \cdots$, and some $j=0,1$, $2, \cdots$, set $v_{n}=\left(l-w_{n+1}\right) /\left(w_{1}-w_{k(i)}\right)$.

Now let $T=v \otimes \lim +B$. By computing $T^{* *}$ it is easily verified that the matrix obtained from $T^{* *}$ by multiplying each of its nondiagonal elements by -1 is the two sided inverse of $T^{* *}$ and is the second adjoint of the operator $-v \otimes \lim +(2 I-B)$. Thus $T^{* *}$ is an isomorphism from $m$ onto $m$ and hence $T$ is an isomorphism in $B[c]$. It remains only to compute $T^{* *} w$. Let $t_{i}$ denote the number of terms in the sequence $w$ between $w_{k(i)}$ and $w_{k(i+1)}$. Then $T^{* *} w=\left(w_{1}, \cdots, w_{k(1)}, l, \cdots, l, w_{k(2)}, l, \cdots, l, w_{k(3)}, l, \cdots\right)$, where $t_{i} l$ 's appear between $w_{k(i)}$ and $w_{k(i+1)}$. Since $\lim _{i} w_{k(i)}=l$ and $l \neq w_{1}$, $T^{* *} w$ clearly belongs to $c \mid \hat{c}$.

8. Lemma. Let $x \in c \mid \hat{c}$. Then there exists an isomorphism $T$ in $B[c]$ such that $T^{* *} x=e^{1}$.

Proof. Suppose first that $x_{1} \neq 0$. Since $x \in c \mid \hat{c}, \lim x$ exists and does not equal $x_{1}$. Let $l=x_{1}-\lim x$. Let $v=\left(-1 /\left(l x_{1}\right)\right)\left(x_{2}, x_{3}, x_{4}, \cdots\right)$ and let $B=\left(b_{n k}\right)$ be the diagonal matrix $b_{n n}=1 / l, n=1,2,3, \cdots$. Then $T=$ $v \otimes \lim +B$ is the isomorphism. Indeed, a simple computation shows that $T^{* *} x=e^{1}$ and that $T^{-1}=u \otimes \lim +A$, where $u=\left(x_{2}, x_{3}, x_{4}, \cdots\right)$ and $A=\left(a_{n k}\right)$ is the diagonal matrix $a_{n n}=l, n=1,2, \cdots$.

Next suppose that $x_{1}=0$. Then $\lim x=l \neq 0$. Let $k>1$ be the first subscript such that $x_{k} \neq 0$. Let $T=v \otimes \lim +B$, where $v=(1 / l) e^{k-1}$ and $B=\left(b_{n k}\right)$ is the matrix defined by

$$
\begin{aligned}
b_{n n} & =-1 / l & & \text { for } n \geqq k, \\
b_{n, k-1} & =x_{n+1} /\left(l x_{k}\right) & & \text { for } n \geqq k, \quad \text { and } \\
b_{n n} & =1 & & \text { for } n=1,2, \cdots, k-2 .
\end{aligned}
$$

(If $k=2$ ignore the last step.) Then $T^{* *} x=e^{1}$ and $T$ is an isomorphism with inverse $T^{-1}=u \otimes \lim +A$, where $u_{n-1}=x_{n}$ for each $n \geqq k$ and where

$$
\begin{aligned}
a_{n n} & =-l & & \text { for } n \geqq k, \\
a_{n, k-1} & =x_{n+1} & & \text { for } n \geqq k-1, \text { and } \\
a_{n n} & =1 & & \text { for } n=1,2, \cdots, k-2 .
\end{aligned}
$$

(Again, ignore the last step if $k=2$.) 
9. TheOREM. Let $w \notin \hat{c}$. Then there exists an isomorphism $T$ in $B[c]$ such that $T^{* *} w=e^{1}$.

Proof. Simply combine the two isomorphisms gotten in Lemmas 7 and 8.

Recall that $\rho_{w}^{\perp}=\left\{T \in \Omega_{w}: \rho_{w}(T)=0\right\}$. $\rho_{w}^{\perp}$ is clearly a subalgebra of $\Omega_{w}$.

10. TheOREM. Let $w \notin \hat{c}$. Then there exists an algebra isomorphism $\phi$ from $B[c]$ onto $B[c]$ such that,

(a) $\Omega_{w}=\phi(\Omega)$,

(b) $\rho_{w}^{\perp}=\phi\left(\rho^{\perp}\right)$, and

(c) $\Gamma_{w}=\phi(\Gamma)$.

Proof. Let $T$ be an isomorphism in $B[c]$ whose second adjoint sends $w$ to $e^{1}$. Define $\phi: B[c] \rightarrow B[c]$ by $\phi(S)=T^{-1} S T$. It is easily verified that $\phi$ is an algebra isomorphism from $B[c]$ onto $B[c]$ with inverse $\phi^{-1}(S)=T S T^{-1}$. It remains only to verify the three assertions. We do this for (a); the proofs for (b) and (c) are similar. Let $S \in \Omega=\Omega_{e^{1}}$. Then $S^{* *} e^{1}=\lambda e^{1}+\hat{x}$ with $\hat{x} \in \hat{c}$. Thus $(\phi(S))^{* *} w=\left(T^{-1} S T\right)^{* *} w=\left(T^{-1} S\right)^{* *} e^{1}=T^{-1 * *}\left(\lambda e^{1}+\hat{x}\right)=$ $\lambda w+T^{-1 * *} \hat{x} \in w \oplus \hat{c}$ and so $\phi(\Omega) \subset \Omega_{w}$. On the other hand, if $S \in \Omega_{w}$ then $S^{* *} w=\mu w+\hat{y}$ for some $\hat{y} \in \hat{c}$, and so $\left(\phi^{-1}(S)\right)^{* *} e^{1}=\left(T S T^{-1}\right)^{* *} e^{1}=$ $(T S)^{* *} w=T^{* *}(\mu w+\hat{y})=\mu e^{1}+T^{* *} \hat{y} \in e^{1} \oplus \hat{c}$. Thus, $\Omega_{w} \subset \phi(\Omega)$.

Let $K$ denote the set of compact operators in $B[c]$.

11. Corollary. $\left\{\Omega_{w}: w \in m\right\}=I \oplus K$.

Proof. If $w \in \hat{c}$ then $\Omega_{w}=B[c]$ (Lemma 3(a)) and so $\bigcap\left\{\Omega_{w}: w \in m\right\}=$ $\bigcap\left\{\Omega_{w}: w \notin \hat{c}\right\}$. For each $w \notin \hat{c}$ we have $\Omega_{w}=\phi(\Omega)$, where $\phi$ is the algebra isomorphism constructed in Theorem 10. But $\Omega=I \oplus \rho^{\perp}$ and so $\Omega_{w}=$ $\phi(\Omega)=I \oplus \phi\left(\rho^{\perp}\right)=I \oplus \rho_{w}^{\perp}$. Hence, $\bigcap\left\{\Omega_{w}: w \in m\right\}=I \oplus \bigcap\left\{\rho_{w}^{\perp}: w \in m\right\}$. Since each $\rho_{w}^{\perp}$ contains $K$ [5, Theorem 3] the proof will be complete when we show that $\bigcap \rho_{w} \subset K$. Thus let $T=v \otimes \lim +B \in \bigcap \rho_{w}^{\perp}$. (Notice that $v \in c$ and that $B \in \Gamma$ because $T \in \rho^{\perp} \subset \Omega$.) Then $T^{* *} w^{*} \in \hat{c}$ for every bounded sequence $w$. This means that $B$ is conull and sums every bounded sequence and hence must be compact by Schur's theorem. (See [4, p. 17].) Since $v \otimes \lim$ is also compact it follows that $T \in K$.

12. ThEOREM. $\bigcap\left\{\Gamma_{w}: w \in m\right\}=\{\lambda I: \lambda$ a scalar $\}$.

Proof. Let $T \in \bigcap \Gamma_{w}$. Then, in particular, $T \in \Gamma$ and so $\chi(T)=\rho(T)$, $\chi_{i}(T)=0(i=1,2, \cdots)$ and $T=\left(t_{n k}\right)$ with column limits $t_{k}(k=1,2, \cdots)$. Let $w \in c \mid \hat{c}$, say $w=\mu e^{1}+\hat{x}$ with $\mu \neq 0$ and $\hat{x} \in \hat{c}$. By computing $T^{* *} w$ and equating it to $\rho_{w}(T) w$ we get the following set of equations:

$$
\sum_{k} t_{k} \hat{x}_{k+1}=0 \quad \text { and } \quad \sum_{k} t_{n k} \hat{x}_{k+1}=\rho_{w}(T) \hat{x}_{n+1} \quad(n=1,2, \cdots) .
$$


Since $\rho$ is the only scalar homomorphism on $\Omega\left(=\Omega_{w}\right)[1], \rho_{w}=\alpha \rho$ for some scalar $\alpha$. But the proof of Lemma 2(a) shows that $\alpha$ must be 1. Hence, $\rho_{w}=\rho$ on $\Omega$ and the latter of the equations becomes

$$
\sum_{k} t_{n k} \hat{x}_{k+1}=\rho(T) \hat{x}_{n+1} \quad(n=1,2, \cdots) .
$$

In particular, taking $\hat{x}=e^{k}(k=2,3,4, \cdots)$ we get $t_{n k}=0$ for $n \neq k$ and $t_{n n}=\rho(T)$. Thus $T=\rho(T) I$ and so $\cap \Gamma_{w}$ is contained in the set $\{\lambda I: \lambda$ a scalar\}. Since the reverse containment is obvious, the proof of the theorem is complete.

Our next result improves [5, Theorem 4].

13. Theorem. $\Gamma_{z}=\Gamma$ if and only if $z=\mu e^{1}$ with $\mu \neq 0$.

Proof. The second half of this theorem is contained in the statement of Lemma 2(b). To prove the first half, assume that $z \neq \mu e^{1}$ for any $\mu \neq 0$. Then $z_{i} \neq 0$ for some $i>1$. Either this is the only nonzero entry in $z$, or else $z_{j} \neq 0$ for some $j$ different from $i$. In the first case, let $T=\left(t_{n k}\right)$ be the matrix with $t_{n, i-1}=1$ for $n=1,2,3, \cdots$, and zeros elsewhere. Then $T^{* *} z=$ $z_{i} e \neq \lambda z$ for any $\lambda$; hence, $T$ belongs to $\Gamma$ but not to $\Gamma_{z}$. In the second case, take $T=\left(t_{n k}\right)$ to be the matrix with $t_{i-1, i-1}=1$ and zeros elsewhere. Then $T^{* *} z=z_{i} e^{i} \neq \lambda z$ for any $\lambda$ and so we again have $T$ in $\Gamma$ but not in $\Gamma_{z}$.

\section{REFERENCES}

1. H. I. Brown, D. R. Kerr and H. H. Stratton, The structure of $B[c]$ and extensions of the concept of conull matrix, Proc. Amer. Math. Soc. 22 (1969), 7-14.

2. H. I. Brown and H. H. Stratton, Some FK spaces that are conservative summability fields, J. London Math. Soc. (2) 3 (1971), 363-370. MR 43 \#5212.

3. J. P. Crawford, Transformations in Banach spaces with applications to summability theory, Ph.D. Dissertation, Lehigh University, Bethlehem, Pa., 1966.

4. G. M. Petersen, Regular matrix transformations, McGraw-Hill, New York, 1966. MR 37 \#642.

5. A. Wilansky, Subalgebras of $B[X]$, Proc. Amer. Math. Soc. 29 (1971), 355-360. MR 43 \#7930.

6. - Topological divisors of zero and Tauberian theorems, Trans. Amer. Math. Soc. 113 (1964), 240-251. MR 29 \#6222.

7. —-, Functional analysis, Blaisdell, New York, 1964. MR 30 \#425.

Department of Mathematics, State University of New York at Albany, Albany, New York 12222 (Current address of H. I. Brown)

Current address (Tae-Geun Cho): Department of Mathematics, Sogang University, Seoul, Korea 